\title{
Comprensión de lectura de artículos científicos en formato digital e impreso en estudiantes de medicina humana
}

\author{
Reading comprehension of digital and printed scientific articles in human \\ medicine students
}

\author{
Hans Contreras-Pulache ${ }^{1}$, Javier Sevillano-Jimenez ${ }^{1}$, Alfonso Rodríguez-Saldarriaga ${ }^{2}$, Jeel \\ Moya-Salazar $^{1,3}$, Arturo Basurto-Ayala ${ }^{1}$ \\ ${ }^{1}$ Escuela Académico Profesional de Medicina Humana, Facultad de Ciencias de la Salud. \\ Universidad Privada Norbert Wiener. Lima, Perú \\ ${ }^{2}$ Concejo Nacional de Ciencia y Tecnología. Concytec, Lima, Perú \\ ${ }^{3}$ Hospital Nacional Docente Madre Niño San Bartolomé, Lima, Perú.
}

\begin{abstract}
Resumen
El avance tecnológico facilita el acceso a la información, así es como logra desplazar a los medios antiguos por su practicidad. En el ambiente educativo se debe evaluar el impacto que tiene la digitalización al leer y comprender un texto ya que la lectura es un acto inherente a la formación académica. Por ello en el presente estudio se busca comparar la comprensión lectora de un artículo científico (carta científica) leído en formato impreso (en papel) y en formato digital en estudiantes de medicina de tercer año de la Universidad Norbert Wiener. Metodología: Estudio experimental aleatorizado en 52 estudiantes (Grupo A 25, Grupo B 27) divido en dos etapas: i) lectura de un artículo impreso y virtual; y ii) intercambio de grupos y lecturas. Se evaluó comprensión de lectura literal y comprensión de lectura inferencial evaluada con cinco preguntas de respuesta múltiple. Resultados: La suma de puntajes obtenidos por modalidad nos muestra que al momento de leer de manera virtual tuvieron una puntuación de 212 y cuando leyeron en forma impresa fue de 228. Los modelos de estimación asocian significativamente la lectura impresa con el puntaje de compresión lectora, así como también con el promedio ponderado; este último es visualizado como un buen predictor de la mayor diferencia en la prueba de compresión lectora. Conclusiones: La lectura impresa y el alto promedio ponderado se asocian con un mayor puntaje en preguntas de comprensión de lectura de un artículo científico en estudiantes de medicina de tercer año de la Universidad Privada Norbert Wiener.
\end{abstract}

Palabras clave: comprensión de lectura, educación médica, Perú, estudiantes de medicina, medios de lectura.

Fecha de envío: 15/11/2020

Fecha de aceptación: 01/01/2021 


\begin{abstract}
Technological advance facilitates access to information, thus managing to displace the old media due to its practicality. In the educational field the impact of digitization should be evaluated when reading and understanding a text since reading is an act inherent to academic training. Therefore, this study seeks to compare the reading comprehension of a scientific article (letter to the editor) read in printed format (on paper) and in digital format in third-year medical students at Norbert Wiener University. Methodology: Randomized experimental study in 52 students (Group A 25, Group B 27) divided into two stages: i) reading a printed and virtual article; and ii) exchange of groups and readings. Literal reading comprehension and inferential reading comprehension were assessed with five questions with multiple answers. Results: The sum of the scores obtained by modality shows us that at the time of reading virtually they had a score of 212 and when they read on paper it was 228 . The estimation models significantly associate printed reading with the reading comprehension score, as well as being the weighted average, the latter seen as a good predictor of the greatest difference in the reading comprehension test. Conclusions: Print reading and high weighted average are associated with higher scores on reading comprehension questions in a scientific article in third-year medical students from Norbert Wiener Private University.
\end{abstract}

Keywords: reading comprehension, medical education, Peru, medical students, reading media.

\section{Introducción}

La lectura es un acto inherente a la formación académica, en especial para el médico en formación, por lo que es importante que el médico desarrolle el hábito de lectura de manera continua, rigurosa y crítica. La lectura, en la actualidad, ha entrado en una especie de contradicción. Esto se puede explicar ya que ahora contamos con todos los medios para facilitar la lectura, en comparación con siglos anteriores, pero no se desarrolla en nuestro país de manera óptima el hábito de leer. (1) Actualmente vivimos en un contexto donde los hábitos de lectura y la capacidad de comprensión lectora están amenazados. Esto es alarmante, y más estando en una época donde gracias al avance de la conectividad y las tecnologías digitales, el acceso al conocimiento es cada vez más sencillo para todo el mundo. Por ello, para un estudiante de medicina en el mundo contemporáneo, el problema ya no es el acceso a trabajos académicos, sino leerlos y comprenderlos adecuadamente (2-4). Por esta razón, que ciertos investigadores han empezado a mostrar interés por comprender si lo digital, como medio de lectura, es o no un facilitador cuando se lee un artículo académico $(5,6)$. Con respecto al tema investigado, aún no existe un consenso debido que están quienes proponen que no habría relación entre el medio de lectura (impreso o digital) y la compresión del lector, así como también hay autores que sugieren que los estudiantes suelen comprender menos cuando leen en formato digital que cuando leen en formato impreso. Debido a los escasos trabajos de investigación en esta índole de procedencia peruana (1), se optó por presentar como objetivo del presente estudio evaluar los hábitos de lectu- 
ra académica y su capacidad de comprensión de lectura de un artículo científico, publicado en una revista académica de prestigio, en estudiantes peruanos de medicina humana. Se consideró dos modos de lectura: digital y lectura impresa.

\section{Materiales $\mathbf{y}$ Métodos}

\subsection{Diseño y población de estudio}

Con el objetivo de saber bajo qué modalidad de lectura los estudiantes muestran un mejor desempeño, si mediante el formato virtual o mediante el formato impreso, se diseñó un estudio experimental aleatorizado sin tratamiento, dado que la variable de interés no requiere ningún tipo de intervención, de corte transversal divido en dos etapas.

Como criterios de inclusión en el estudio, los participantes debían estar cursando el semestre 2019-II del tercer año, haber completado correctamente todos los datos de la ficha de recolección de datos inicial y haber firmado y estar de acuerdo con la participación en el presente estudio. Se excluyó aquellos participantes que hayan adelantado algún curso, así como también a aquellos que no completaron en su totalidad o de manera incorrecta la ficha de recolección, así como a aquellos que no deseaban participar en el estudio.

Luego de la selección de participantes, la población del estudio fue conformada por 52 estudiantes (aleatorizados en dos grupos de 25 y 27 estudiantes, grupo A y B respectivamente. Se diseñó un ensayo de tipo cruzado, donde los estudiantes fueron su propio grupo de control) que cursaban el tercer año de la carrera de medicina humana en la Universidad Privada Norbert Wiener, en Lima, Perú durante el
2019-II. La aleatorización definió, en la primera etapa, a los estudiantes que se les asignó la lectura en formato virtual y a los que se les asignó la lectura en formato impreso. Luego se hizo un descanso de 5 minutos y se prosiguió con la segunda etapa. En esta, se intercambiaron los grupos: los que en una primera etapa leyeron en formato virtual y pasaron a leer una segunda lectura en formato impreso, y viceversa (7-9).

La asignación aleatoria se hizo teniendo en cuenta el balance de las variables edad, sexo y el resultado de una prueba cognitiva tomada previamente (que incluía preguntas liberadas de la prueba PISA). La cantidad de individuos en cada grupo es muy similar (27 y 25 ). Por ello, en un primer momento, pudo inferirse que la muestra hay estado balanceada en las variables explicativas.

\subsection{Selección de las lecturas}

Para la medición del nivel de compresión lectora en artículos científicos, se necesitó elegir producción científica que no tuviera una gran extensión. Para la selección de las lecturas científicas que se utilizaron en el presente estudio, se tuvo como criterios que el artículo científico sea de producción reciente (no mayor a 5 años), también que la lectura no sea muy extensa (no mayor a 2 hojas), y a su vez que el articulo cuente con una o más tablas que muestren sus resultados. Estas características se precisaron para facilitar la lectura integral del documento en un breve periodo de tiempo (el periodo de duración de la prueba), sobre todo considerando que el proceso se repetiría dos veces (dos lecturas). Los documentos académicos más idóneos para con estas exigencias fueron las cartas científi- 
cas (cuya extensión máxima era de 800 palabras); y se procedió a seleccionar dos (entre las más leídas o citadas), dentro de las que estuvieran publicadas en una revista seria y reconocida (Revista peruana de medicina experimental y salud pública).

\subsection{Intervención de lectura}

El tiempo de lectura en cada etapa fue de 15 minutos. Este tiempo incluyó la resolución de un breve cuestionario para evaluar comprensión de lectura. Para la aleatorización de los grupos y para mantenerlos lo más homogéneos posible $(7,8)$, se utilizó una ficha de recolección de datos, donde se buscaba recolectar la edad, sexo, antecedente de haber repetido un curso, promedio ponderado, número de artículos (o papers) leídos en el curso del último año, y hobbies relacionados con la literatura académica o la lectura en general. Además de ello, se aplicó un cuestionario con preguntas liberadas de la prueba PISA (Programme for International Student Assesment) con el que se buscó medir el nivel de capacidad lectora del estudiante antes de realizar la intervención. Para la intervención se utilizaron dos cartas científicas, las cuales fueron publicadas en la RPMESP. La primera carta (10) fue publicada el año 2018 con el título "¿Son menos dañinas las gaseosas sin azúcar? Un análisis de la información nutricional en dos ciudades peruanas" y la segunda carta (11), en el año 2017 con el título "Análisis de micromatrices cromosómicas en niños peruanos con retraso del desarrollo psicomotor o discapacidad intelectual". Para una mejor explicación los procederemos a llamar "Artículo 1" y "Artículo 2", respectivamente.

Un día previo a la intervención se registra- ron los datos de los participantes a través de la ficha de recolección de datos. Además, se les pidió firmar un consentimiento informado donde se les brindó toda la información sobre el tratamiento de los datos obtenidos mediante el estudio, además de ello se les resolvió todas las dudas sobre su participación en el estudio. El experimento se dividió en dos etapas:

I. Primera etapa (lectura del Artículo 1): el grupo A empezó con la lectura digital en una sala de cómputo, mientras que el grupo B inició con la lectura en formato impreso en un salón de clase de la universidad. La prueba consistió en la lectura del Artículo 1.

II. Segunda etapa (lectura del Artículo 2): se volvió a repetir la intervención, pero con los medios de lectura intercambiados: el grupo A leyó la versión impresa y el grupo B la versión digital del Artículo 2.

Luego de la lectura de ambos artículos, se procedió a tomar un cuestionario breve para evaluar la comprensión lectora. Dicho cuestionario comprendió un total de cinco preguntas con respuesta múltiple (se evaluó comprensión de lectura literal e inferencial) $(12,13)$. Estas preguntas fueron realizadas y revisadas por los autores, además de contar con la ayuda de personal con experiencia en docencia, investigación y mentoría en educación científica, para la revisión crítica y corrección del cuestionario. Sumado a ello, los instrumentos de evaluación de comprensión lectora fueron revisados y aprobados por un comité de expertos externos; estos profesionales se dedican a la investigación y la docencia universitaria, siendo en total tres jueces. 


\subsection{Consideraciones éticas}

El proyecto de investigación del presente trabajo se presentó al fondo concursable de la Universidad Privada Norbert Wiener. Pasó por los comités metodológico y ético y recibió la aprobación correspondiente para su ejecución. Todos los estudiantes evaluados fueron informados de la anonimidad de su participación tanto como de su voluntariedad. Se recalcó que no habría ninguna acción sobre aquellos que no desearan participar. La participación en esta investigación no vulneró los principios éticos (no maleficencia, beneficencia, autonomía y justicia).

\subsection{Variables y análisis de datos}

Las variables que se consideraron en este estudio se agrupan en las siguientes dimensiones:

- Variables de antecedentes socioeconómicos (sexo, edad, institución dónde culminó su secundaria, trabajo).

- Variables de educación (repitió un año en primaria o secundaria, repitió un curso en la universidad, carrera previa, promedio ponderado en la universidad, capacidad lectora).

- Variables de hábitos de lectura científica (número de artículos leídos en el último año).

- Variables de uso del tiempo (hobbies).

- Variables de comprensión de lectura (cuestionario para el Artículo 1 y 2).

Para la recolección, gestión y análisis de datos se elaboró una ficha de recolección de datos con todas las variables del estudio. Se utilizaron tres métodos para el análisis de los resultados. El primero fue la comparación entre las medias obtenidas en la evaluación final de comprensión lectora. El segundo, el desarrollo de un análisis de las distribuciones de los resultados, separados por grupos, para poder evaluar si existen diferencias entre los demás momentos de la distribución aparte de la media. El tercer método incluyo un análisis multivariado con variables de control como sexo, edad, y el promedio ponderado de notas con tal de obtener coeficientes de estimación más eficientes (el error estándar (E.S.) se reduce al incluir variables de control). Se siguieron los métodos de Wooldridge (2002), Rogers \& Hopkins (1988), Cohen (1977/1988) y Greene (2000) desarrollados de manera estándar en los análisis econométricos (14-17). El análisis de datos se realizó en el procesador estadístico STATA v14.0 (StataCorp, Lakeway, Texas) para Linux (LinuxFoundation, San Francisco, EE.UU.).

\section{Resultados}

La edad promedio para la muestra global

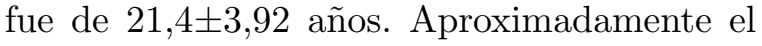
$80 \%$ fueron mujeres y $\sim 40 \%$ tuvieron antecedente de haber repetido algún curso en la universidad. La media del promedio ponderado de notas fue de $13,7 \pm 1$. Solo el $40 \%$ tuvo algún hobby relacionado a la lectura, y el promedio de papers leídos en el último año fue 11 \pm 1 . (Tabla 1)

Los puntajes obtenidos por los estudiantes del Grupo A para el Artículo 1 (modalidad de lectura digital) tuvieron una media de 4,60 (un puntaje mínimo de 2 y máximo de 7 ), dan- 
Contreras-Pulache et al.

\begin{tabular}{|l|c|c|c|c|c|c|c|c|c|}
\hline \multirow{2}{*}{ Variable } & & \multicolumn{2}{|c|}{ Total $\mathbf{( n = 5 2 )}$} & \multicolumn{2}{c|}{ Grupo A $\mathbf{( n = 2 5 )}$} & Grupo B (n=27) & \multicolumn{2}{c|}{ Diferencia } \\
\cline { 2 - 11 } & Dummy & $\mathrm{X}$ & $\mathrm{DE}$ & $\mathrm{X}$ & $\mathrm{DE}$ & $\mathrm{X}$ & $\mathrm{DE}$ & Coef. & std. Err. \\
\hline Edad & & 21,42 & 3,92 & 21,56 & 4,37 & 21,30 & 3,54 & $-0,264$ & $(1,108)$ \\
\hline Sexo & $\mathrm{D}$ & 0,79 & 0,41 & 0,76 & 0,44 & 0,81 & 0,40 & 0,0548 & $(0,116)$ \\
\hline $\begin{array}{l}\text { Repitió } \\
\text { curso }\end{array}$ & $\mathrm{D}$ & 0,37 & 0,49 & 0,40 & 0,50 & 0,33 & 0,48 & $-0,0667$ & $(0,136)$ \\
\hline $\begin{array}{l}\text { Capacidad } \\
\text { lectora } \\
\text { (PISA) }\end{array}$ & & 3,13 & 1,25 & 3,36 & 1,32 & 2,93 & 1,17 & $-0,434$ & $(0,347)$ \\
\hline $\begin{array}{l}\text { Promedio } \\
\text { pondera- } \\
\text { do }\end{array}$ & & 13,68 & 1,09 & 13,77 & 1,04 & 13,59 & 1,14 & $-0,173$ & $(0,303)$ \\
\hline Hobby & $\mathrm{D}$ & 0,38 & 0,49 & 0,48 & 0,51 & 0,30 & 0,47 & $-0,184$ & $(0,136)$ \\
\hline Papers & & 12,04 & 0,95 & 11,92 & 0,81 & 12,15 & 1,06 & 0,228 & $(0,261)$ \\
\hline
\end{tabular}

Tabla 1: Características de los estudiantes de medicina incluidos en el modelo de comprensión lectora.

Abreviaturas: X: promedio, DE: desviación estándar. Papers: artículos científicos leídos el último año previo a la evaluación.

do una suma total en el Artículo 1 de 115. Para el Artículo 2 (modalidad de lectura impresa) se obtuvo una media de 3,76 (un puntaje mínimo de 2 y máximo de 6 ), y se obtuvo una suma total de puntuaciones de 94 . En el caso de los estudiantes del Grupo B, se obtuvo que para el Artículo 1 (modalidad de lectura impresa) tuvieron una media de puntuación de 4,96 (un puntaje mínimo de 2 y máximo de 7), obteniendo una suma total del Grupo para el Artículo 1 de 134; y para el Artículo 2 (modalidad de lectura digital) se obtuvo una media de 3,59 (un puntaje mínimo de 2 y máximo de 6), obteniendo una suma total de Grupo para el Artículo 2 de 97. Estos resultados se muestran en la Tabla 2.

Con los puntajes finales de cada grupo, acorde a su método de lectura, se elaboró la Tabla 3, donde se evalúan los puntajes obtenidos según modalidad de lectura. Se tiene que la lectura en modalidad virtual tuvo una media de 4,08 (un mínimo de 2, un máximo de 7 y una suma total de 212); por otro lado, se evidencia que la lectura en modalidad impresa tuvo una media de 4,38 (un puntaje mínimo de 2 y máximo de 7 , obteniendo una suma total de 228).

Como se puede evidenciar, cuando los estudiantes leyeron de forma impresa se observó un incremento de puntuación en la prueba de comprensión de lectura; es decir, en apariencia, los estudiantes que leyeron el documento científico de modo impresa tienden a obtener mayores puntajes en comprensión de lectura. Uno de nuestros modelos considera (ver Tabla 4), además, que cuanto mayor es el promedio ponderado, mayor también será la probabilidad de obtener puntaje más alto en la prueba de comprensión de lectura.

Se muestra a continuación el resultado de 


\begin{tabular}{|c|c|c|c|}
\hline \multirow{2}{*}{\multicolumn{2}{|c|}{ Grupos }} & \multicolumn{2}{|c|}{ Puntaje $^{a}$} \\
\hline & & \multirow{2}{*}{$\begin{array}{c}\text { Artículo } \mathbf{1} \\
4,60\end{array}$} & \multirow{2}{*}{$\begin{array}{c}\text { Artículo 2 } \\
3,76\end{array}$} \\
\hline \multirow{4}{*}{ Grupo $A^{*}$ (25 integrantes) } & Media & & \\
\hline & Mínimo & 2 & 2 \\
\hline & Máximo & 7 & 6 \\
\hline & Suma & 115 & 94 \\
\hline \multirow{4}{*}{ Grupo $B^{* *}$ (27 integrantes) } & Media & 4,96 & 3,59 \\
\hline & Mínimo & 2 & 2 \\
\hline & Máximo & 7 & 6 \\
\hline & Suma & 134 & 97 \\
\hline
\end{tabular}

Tabla 2: Análisis de Frecuencias de las notas obtenidas en controles de lectura por Grupo aleatorizado. ${ }^{a}$ Puntaje mínimo posible: 0; Puntaje máximo posible: 8. * Artículo 1: Evaluación de lectura en digital; Artículo 2: Evaluación de lectura en Físico. ${ }^{* *}$ Artículo 1: Evaluación de lectura en Físico; Artículo 2 : Evaluación de lectura en digital.

\begin{tabular}{|l|l|c|}
\hline \multirow{4}{*}{ Lectura digital (52 participantes) } & Media & 4,08 \\
\cline { 2 - 3 } & Mínimo & 2 \\
\cline { 2 - 3 } & Máximo & 7 \\
\cline { 2 - 3 } & Suma & 212 \\
\hline \multirow{4}{*}{ Lectura impresa (52 participantes) } & Media & 4,38 \\
\cline { 2 - 3 } & Mínimo & 2 \\
\cline { 2 - 3 } & Máximo & 7 \\
\cline { 2 - 3 } & Suma & 228 \\
\hline
\end{tabular}

Tabla 3: Notas obtenidas en los controles de lectura según modalidad de lectura (impreso vs digital).

estimar el modelo para cada prueba por separado. (Tabla 4) Las estimaciones muestran resultados consistentes entre sí, aunque los efectos tengan tamaños distintos y no sean significativamente distintos de cero en todos los casos. La variable de agrupación es una variable binaria que señala a los estudiantes que leyeron en modalidad digital o impresa $(0=$ digital; 1 = impresa). Para probar la consistencia del modelo, se hicieron regresiones independientes con la variable capacidad de lectura (PISA) y la variable de promedio ponderado (ambas son mediciones anteriores del logro académico o cognitivo alcanzado).

La Tabla 5 muestra el resultado de estimar el modelo para la diferencia de la nota obtenida entre la primera y segunda etapa en un mismo individuo. Como se puede ver, la modalidad de lectura impresa se asocia significativamente con la modalidad de lectura impresa y con el promedio ponderado $(\mathrm{p}<0,05)$; siendo el efecto mayor (y más del doble) del primero (lectura impresa) con respecto al segundo (promedio ponderado). 
Contreras-Pulache et al.

\begin{tabular}{|c|c|c|c|c|c|c|c|c|}
\hline \multirow{3}{*}{ Variable } & \multicolumn{4}{|c|}{ Estimaciones para la primera prueba } & \multicolumn{4}{|c|}{ Estimaciones para la segunda prueba } \\
\hline & \multicolumn{2}{|c|}{ Grupo (A) } & \multicolumn{2}{|c|}{ Grupo (B) } & \multicolumn{2}{|c|}{ Grupo (A) } & \multicolumn{2}{|c|}{ Grupo (B) } \\
\hline & Coef. & ES & Coef. & ES & Coef. & ES & Coef. & ES \\
\hline $\begin{array}{l}\text { Medio de } \\
\text { Lectura }\end{array}$ & 0,57 & $-0,379$ & $0,843^{* *}$ & $-0,389$ & 0,118 & $-0,364$ & 0,167 & $-0,372$ \\
\hline Edad & $-0,00377$ & $-0,0586$ & $-0,00458$ & $-0,0441$ & 0,0267 & $-0,0687$ & 0,0102 & $-0,0713$ \\
\hline Sexo & 0,476 & $-0,454$ & 0,233 & $-0,484$ & 0,278 & $-0,463$ & 0,282 & $-0,466$ \\
\hline $\begin{array}{l}\text { Repitió } \\
\text { curso }\end{array}$ & $-0,369$ & $-0,424$ & 0,48 & $-0,524$ & $-0,0915$ & $-0,342$ & $-0,0871$ & $-0,438$ \\
\hline $\begin{array}{l}\text { Capacidad } \\
\text { lectora } \\
(\text { PISA) }\end{array}$ & $-0,0725$ & $-0,16$ & & & 0,157 & $-0,113$ & & \\
\hline $\begin{array}{l}\text { Promedio } \\
\text { pondera- } \\
\text { do }\end{array}$ & & & $0,557^{* *}$ & $-0,208$ & & & 0,0732 & $-0,244$ \\
\hline Hobby & 0,24 & $-0,413$ & 0,309 & $-0,402$ & $-0,0408$ & $-0,327$ & $-0,0934$ & $-0,337$ \\
\hline Constante & $4,228^{* *}$ & $-1,726$ & $-3,942$ & $-3,512$ & 2,243 & $-1,54$ & 2,054 & $-3,551$ \\
\hline $\begin{array}{l}\text { Observa- } \\
\text { ciones }\end{array}$ & \multicolumn{2}{|c|}{52} & \multicolumn{2}{|c|}{52} & \multicolumn{2}{|c|}{52} & \multicolumn{2}{|c|}{52} \\
\hline $\begin{array}{l}\text { R- } \\
\text { squared }\end{array}$ & \multicolumn{2}{|c|}{0,138} & \multicolumn{2}{|c|}{0,243} & \multicolumn{2}{|c|}{0,08} & \multicolumn{2}{|c|}{0,056} \\
\hline
\end{tabular}

Tabla 4: Estimaciones entre la primera y segunda prueba de comprensión de lectura en estudiantes de medicina humana.

** $\mathbf{p}<\mathbf{0 , 0 1}$. Abreviaturas:: ES: error estándar; Coef: coeficiente de variación. Papers: artículos científicos leídos el último año previo a la evaluación.

\section{Discusión}

El presente estudio comparó, en estudiantes universitarios de la carrera de Medicina $\mathrm{Hu}-$ mana, el nivel de comprensión de lectura obtenido luego de leer un artículo científico (carta científica) en formato virtual y en formato impreso. Nuestros resultados sugieren que los mejores puntajes de comprensión de lectura están asociados positivamente con el hecho de leer el artículo científico en formato impreso, frente a la lectura en formato digital. Otra variable predictora de un mejor puntaje es, en menor medida, el promedio ponderado del estudiante. Como es de esperar, las variables utilizadas para el balance de la muestra durante la aleatorización presentan coeficientes no significativos en todas las estimaciones. Variables como el antecedente de curso repetidos o la capacidad lectora (evaluada con preguntas de la prueba PISA) o presencia del hobbie asociado a la lectura resultaron no significativas. El modelo pudo explicar un total del $24 \%$ de la varianza según el coeficiente de bondad de ajuste ( $R$ squared). 


\begin{tabular}{|c|c|c|}
\hline & \multicolumn{2}{|c|}{ Prueba } \\
\hline Variables & Coef. & ES \\
\hline Lectura impresa & $1,010^{*}$ & $(0,523)$ \\
\hline Edad & $-0,0148$ & $(0,0651)$ \\
\hline Sexo & $-0,0486$ & $(0,559)$ \\
\hline Repitió curso & 0,567 & $(0,467)$ \\
\hline Capacidad lectora (PISA) & $-0,230$ & $(0,163)$ \\
\hline Promedio ponderado & $0,484^{* *}$ & $(0,233)$ \\
\hline Hobby & 0,403 & $(0,517)$ \\
\hline Constante & $-6,163^{*}$ & $(3,548)$ \\
\hline Observaciones & \multicolumn{2}{|c|}{52} \\
\hline R-Squared & \multicolumn{2}{|c|}{0,24} \\
\hline
\end{tabular}

Tabla 5: Estimaciones de la diferencia entre pruebas de evaluación de compresión lectora en estudiantes de medicina.

${ }^{*} \mathbf{p}<\mathbf{0 , 0 5}{ }^{* *} \mathbf{p}<\mathbf{0 , 0 1}$. Abreviaturas:: ES: error estándar, Coef: coeficiente de variación, Papers: artículos científicos leídos el último año previo a la evaluación.

La vinculación consistente entre la lectura en formato impreso con un alto puntaje en compresión lectora podría deberse a que la lectura impresa permitiría una mejor interacción entre el artículo científico y el estudiante. Sin embargo, nuestros resultados contrastan en específico con lo planteado de modo general por Espinoza \& Gallegos, (18) quienes ponen en evidencia la preferencia por la lectura digital a través de las nuevas tecnologías como computadoras, laptops o smartphones. Esta preferencia por los medios digitales hace que las inversiones en los medios impresos disminuyan. En esta línea generalista también se tiene a Martínez-Fernández et al. (19), quienes evidencian la precaria situación de los medios impresos en papel debido a la reducida inversión en publicidad en medios impresos. En vista a ello, los autores estimaron que el año 2020 sucedería el punto crítico de ruptura de la prensa tradicional, ya que, en entor- nos académicos, la prensa en papel solo representará un mero mercado residual. Por otro lado, Etcheverry (20) señala con respecto a los eBooks que existe un mercado con una demanda en crecimiento y que estos están siendo adoptados gradualmente por los nuevos lectores. Es decir, estamos en un contexto donde todo se rinde a la disrupción digital (21); y en nuestro caso particular de investigación, estudiantes que leen mejor cuando lo hacen en medios impresos y no digitales. Esta situación no solo resulta paradigmática, sino hasta contradictoria, y, sobre todo, dado que se trata de estudiantes universitarios de una de las carreras de mayor impacto social (medicina humana): un riesgo que debe asumirse para su remediación inmediata.

Con respecto a ello, Fuentes, Ana y colaboradores (22) nos mencionan que esta variación puede deberse a la familiarización que tiene el evaluado con el dispositivo tecnológico en el 
que realiza la lectura. En su investigación evaluó la comprensión lectora con el soporte de un ordenador, tablet o celular y papel (impreso), además de ello evaluaron la familiaridad que los estudiantes tienen con los dispositivos. Los autores concluyeron que los jóvenes universitarios evaluados son sensibles al soporte en el que leen, así obtuvieron de forma global que el ordenador fue el mejor soporte para la lectura, seguido por el papel, y el teléfono móvil o tablet. La diferencia que mencionan sobre el ordenador y el teléfono móvil o tablet es que el móvil se usa en su mayoría para el acceso a redes sociales; esto puede influir en la concentración del lector al momento de usar estos medios. Por otro lado, el ordenador, además de contar con una pantalla más amplia, es visto por el estudiante como un medio de educación y un anexo de la universidad, de ese modo, la asociación que tiene el ordenador con el estudio logra que el estudiante tenga un mejor rendimiento en la compresión, a pesar que también tenga acceso a las redes sociales.

En correlación con lo anterior, Wu (23) también realizó pruebas de compresión lectora en soportes físicos e impreso. De ello evidenció que las habilidades de navegación y el uso día a día del internet presentan una asociación positiva directa con las habilidades de navegación y desempeño en las pruebas de lectura.

Por otra parte, para el año 1975 el conocimiento se duplicaba cada 12 años; en 1995 cada 10 años y medio; para el año 2005 cada 5 años; y para el 2016 se daba cada dos años (en ingeniería genética, química e informática, etc.). A partir de esto, Martínez-Fernández \& Castellanos-García estimaron que el 2020 estas mismas áreas se duplicarían cada 73 días (19). La medicina humana, como se entiende, no vive ajena a este contexto dinámico; to- do lo contrario: representa uno de los ámbitos en donde más se investiga y en donde más se actualiza el conocimiento. En este contexto, nuestros resultados revelan un potencial espacio de conflicto, pues habría datos suficientes para sugerir que, aun cuando es bajo el vínculo entre un estudiante de medicina humana y la práctica de la lectura académica y la lectura en general, que los estudiantes que están migrando a preferir lecturas en entornos digitales (que parece ser la norma contemporánea), estos leen mejor cuando lo hacen en formato impreso y no en formato virtual.

Nuestro estudio es uno de los pocos llevados a cabo en estudiantes de medicina que evalúan la comprensión de lectura comparando dos modos de lectura. En este sentido, esta investigación presenta, para nuestra comunidad, un carácter exploratorio. Otros autores, trabajando con un público completamente diferente al nuestro, han llegado a conclusiones contrarias. Dundar y Akcayir (24) realizaron un experimento con estudiantes de quinto año de primaria donde evaluaron la velocidad de lectura y la comprensión lectora mediante la comparación de veinte niños leyendo en una tablet y en un libro impreso. Aleatorizaron dos grupos, donde diez estudiantes fueron del grupo de control y leyeron un libro impreso, y diez del grupo tratado que leyeron por medio de una tablet. En el estudio se evidenció que los resultados tanto para la velocidad de lectura y para la comprensión lectora no fueron significativos en su diferencia entre el medio impreso y digital. Estudios en población universitaria no han demostrado diferencias significativas (25) al momento de comparar la lectura digital con la lectura impresa. Ramalingam et al. (2018) evidenció un ligero incremento en el rendimiento de los estudiantes con medio di- 
gitales (6). Urgen más investigaciones en este campo a fin de encontrar el mejor modo de optimizar y potenciar la formación de un estudiante de medicina humana en términos de investigación científica.

Finalmente, se puede mencionar que la generación actual está dejando de lado los soportes antiguos como la impresión y el libro por los medios digitales, y aunque si bien es cierto, los libros aún son considerados medios de referencia y consulta. Como mencionan los autores Burin y colaboradores (26), los alumnos de hoy forman parte de una generación que prefieren utilizar los recursos digitales, pero sobre recalcan que su uso no es netamente académico, sino que, por el contrario, los llamados "nativos digitales" no utilizan de forma eficiente los medios digitales para fines educativos o laborales, sino que lo utilizan en su mayoría para entretenimiento y redes sociales. Por ello, los autores sostienen que los estudiantes deben desarrollar competencias digitales que comprenden habilidades técnicas, operacionales y cognitivas sobre búsquedas, navegación, integración del conocimiento y evaluación del contenido.

Nuestros resultados deben de interpretarse bajo la limitación de la validez interna, puesto que nuestros hallazgos, en el mejor de los casos, alcanzan a representar a la muestra que se ha estudiado. El mérito esencial de nuestro estudio radica en su sentido de oportunidad, en su carácter primario, y en la naturaleza experimental de su diseño. Aunque aspectos como el tamaño de la muestra y la estandarización de los instrumentos o de los métodos de evaluación de las variables son perfectibles, resulta difícil desde ya resaltar lo complicado que es, en lo operativo, evaluar la comprensión de lectura académica, puesto que casi de ordina- rio cuando se ha evaluado la comprensión de lectura se ha hecho solo en términos de la educación básica y no de la educación superior, y menos de la educación superior en medicina humana (27).

\section{Conclusiones}

De los resultados obtenidos, es posible concluir (al menos para la muestra que se ha considerado) que los estudiantes comprenden mejor un conjunto de ideas (científicas) al leerlas en una versión impresa; esto a contracorriente de las tendencias actuales que tienden a llevar toda actividad académica hacia el mundo digital. Es probable que esto ocurra porque el documento impreso habilita una mayor interacción entre el estudiante y la lectura, que en el caso de ser una lectura académica obliga al subrayado, a la toma de notas, entre otras estrategias. Nuestros resultados buscan de este modo llamar la atención de los educadores o tomadores de decisión en materia educativa, haciendo notar que los ejercicios de lectura pasan por una dinámica particular, por lo que ameritan ser tratados de un modo específico en cada caso. Usualmente, al hablar de lectura se hace referencia a la lectura alfabética y a la lectura aplicada a la educación básica regular.

Somos uno de los pocos trabajos aplicados a evaluar lectura académica, específicamente en el campo de la llamada "literatura médica". Si bien nuestras limitaciones y sesgos restringen la expansión e inferencia de nuestras conclusiones, creemos tener el mérito de ser una de las pocas $-\mathrm{y}$ primeras - investigaciones que se están planteando el tema de la lectura académica como base del ejercicio del trabajo universitario en el caso de la carrera de me- 
Contreras-Pulache et al.

dicina humana. Es necesario ampliar el espectro del estudio y asignar tratamientos distintos para evaluar la variabilidad del fenómeno explorado, siempre reconociendo que la lectura académica es uno de los pilares de toda formación médica.

\section{Referencias}

(1) Santisteban S, Vallejo N. Los Hábitos de Lectura y la Comprensión lectora en Estudiantes Universitarios de la Especialidad de Lengua, Literatura, Psicología y Filosofía en el Año 2014. Rev. Investig. Altoandin. 2015; 17 (3): 397-386.

(2) Rodríguez, A; Ferrer, C; Reina, R; Chinea, B; Fernández, E. Habilidades investigativas en estudiantes de medicina. Universidad de Ciencias Médicas de Sancti Spíritus. Curso 2013-2014. Gaceta Médica Espirituana. 2015. Vol. 17(3).

(3) Bullock A. Does Technology Help Doctors To Access, Use And Share Knowledge? Med Educ. 2014 Jan;48(1):28-33. doi: 10.1111/ medu.12378.

(4) Boruff JT, Storie D. Mobile Devices In Medicine: A Survey Of How Medical Students, Residents, And Faculty Use Smartphones And Other Mobile Devices To Find Information. J Med Libr Assoc. 2014 Jan;102(1):22-30. doi: 10.3163/1536-5050.102.1.006.

(5) Alizadeh I. Medical Students' Perception of Using Electronic Learning Tools in an ESP Program. IJREE 2018; 3(1): 11-18. DOI: 10.29252/ijree.3.1.11
(6) Ramalingam Y, Naid RS, Hariish G, Naidoo JP. Screen Reading vs Paper Reading: An Experimental Study on the Impact of Different Reading Materials on Recall and Comprehension Among Students. Am J Edu Sci. 2018; 4(4): 136143.

(7) Hedges L, Hedberg E. Intraclass Correlation Values for Planning GroupRandomized Trials in Education. Educ Eval Policy Anal. 2007;29(1):60-87. DOI: $10.3102 / 0162373707299706$.

(8) Raudenbush S. Statistical Analysis And Optimal Design For Cluster Randomized Trials. Psycho Met. 1997; 2(2): 173-185.

(9) Sobel M. Identification of Causal Parameters in Randomized Studies with Mediating Variables. J Educ Beah Stat. 2007; 33(2): 230-51. DOI: 10.3102/1076998607307239

(10) Azañedo D, Saavedra-Garcia L, BazoAlvarez JC. ¿son menos dañinas las gaseosas sin azúcar? Un análisis de la información nutricional en dos ciudades peruanas. Rev Per Med Exp Salud Publica. 2018; 35(1):164-66. DOI: 10.17843/rpmesp.2018.351.3555

(11) Abarca-Barriaga HH, Chávez-Pastor MA, Trubnykova M, Vásquez F, Poterico JÁ. Análisis de micromatrices cromosómicas en niños peruanos con retraso de desarrollo psicomotor o discapacidad intelectual. Rev Per Med Exp Salud Publica. 2017; 34(3): 572-74. DOI: 10.17843/rpmesp.2017.343.2741 
(12) Suhadi R. Exploring Literal and Inferential Reading Comprehension On Eightgrade Students, [Tesis] Cirebon: English Language Teaching Department, Terbiyah and Teacher Training Faculty, Syekh Nurjati State Islamic Institute; 2016.

(13) Basaraba D, Yovanoff P, Alonzo J, Tindal G. Examining The Structure Of Reading Comprehension: Do Literal, Inferential, And Evaluative Comprehension Truly Exist? Read Writ. 2013; 26: 349-379. DOI: 10.10007/s11145-012$9372-9$

(14) Wooldridge J. Econometric Analysis of Cross Section and Panel Data. 1st. Ed. London: The MIT Press Cambridge; 2002 .

(15) Rogers W, Hopkins K. Power Estimates in the Presence of a Covariate and Measurement Error. Educ Psyc Measurement. 1988; 48(3):647-56.

(16) Cohen J. Statistical Power Analysis For The Behavioral Sciences. New York: Academic Press; 1977

(17) Cohen, J. (1988). Statistical Power Analvsis For The Behavioral Sciences. 2nd ed. Hillsdale: Lawrence Erlbaum Associates Press; 1988.

(18) Espinoza M, Gallegos D. Preferencias y hábitos en el uso de libros electrónicos y lectura digital. ESPACIOS. 2018; 39(43): 36-47.

(19) Martínez-Fernández V, CastellanosGarcía P, Juanatey-Boga Ó. Del papel a los metamedios: la prensa ante el fin del ciclo impreso. Profesional Información. 2016; 25(3): 331-40-

(20) Etcheverry E. eBooks: escenario actual y tendencias [Tesis de Maestría]. Buenos Aires: Universidad de San Andrés; 2015.

(21) Denegrí MA. Polimatía. Lima: Fondo editorial de la Universidad Inca Garcilazo de la Vega; 2014.

(22) Fuentes A, Jiménez V, Alvarado J. Comprensión lectora digital vs. tradicional según familiaridad con las TIC. Eur. J. Develop. Educa. Psychop. 2020; 8(1): $57-64$

(23) Wu, J-Y. Gender Differences In Online Reading Engagement, Metacognitive Strategies, Navigation Skills And Reading Literacy. Journal of Computer Assisted Learning. 2014; 30, 252-271.

(24) Dundar H, Akcayir M. Tablet vs. Paper: The Effect on Learners' Reading Performance. Int Elect J Elem Educ. 2012; 4(3): 441-450.

(25) Singer LM, Alexander PA. Reading Across Mediums: Effects of Reading Digital and Print Texts on Comprehension and Calibration. J Exper Educat. 2017; 85(1):155-172. DOI: 10.1080/00220973.2016.1143794

(26) Burin D, Coccimiglio Y, González F, \& Bulla J. Desarrollos recientes sobre habilidades digitales y comprensión lectora en entornos digitales. 2016; 6(1), 191- 206. Recuperado de http://revista.psico.edu.uy/index.php/ revpsicologia 
(27) Moya-Salazar J, Rojas-Zumaran V. Tendencias en la investigación del Virus de Papiloma Humano en Latinoamérica y en los en los países de altos ingresos. Rev Col Obst Gin. 2017; 68(3):128-134.

DOI: $10.18597 /$ rcog.2679 